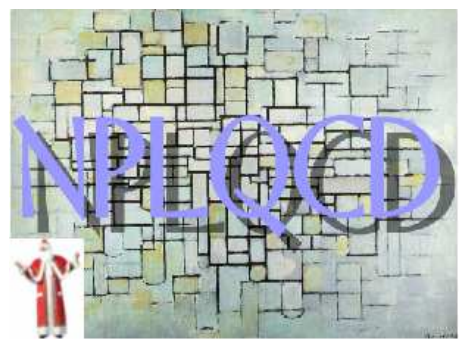

UNH-06-07

UCRL-JRNL-226919

UMD-40762-364

JLAB-THY-06-600

NT@UW-06-031

UG-06-10

\title{
Hyperon-Nucleon Scattering from Fully-Dynamical Lattice QCD
}

\author{
Silas R. Beane, ${ }^{1}$ Paulo F. Bedaque, ${ }^{2}$ Thomas C. Luu ${ }^{3}$ Kostas Orginos, ${ }^{4,5}$ \\ Elisabetta Pallante, ${ }^{6}$ Assumpta Parreño, ${ }^{7}$ and Martin J. Savage ${ }^{8}$ \\ (NPLQCD Collaboration) \\ ${ }^{1}$ Department of Physics, University of New Hampshire, Durham, NH 03824-3568. \\ ${ }^{2}$ Department of Physics, University of Maryland, College Park, MD 20742-4111. \\ ${ }^{3} N$ Division, Lawrence Livermore National Laboratory, Livermore, CA 94551. \\ ${ }^{4}$ Department of Physics, College of William and Mary, Williamsburg, VA 23187-8795. \\ ${ }^{5}$ Jefferson Laboratory, 12000 Jefferson Avenue, Newport News, VA 23606. \\ ${ }^{6}$ Institute for Theoretical Physics, University of Groningen, Nijenborgh 4, \\ 9747 AG Groningen, The Netherlands. \\ ${ }^{7}$ Departament d'Estructura $i$ Constituents de la Matèria, Universitat de Barcelona, \\ E-08028 Barcelona, Spain. \\ ${ }^{8}$ Department of Physics, University of Washington, Seattle, WA 98195-1560.
}

\begin{abstract}
We present results of the first fully-dynamical lattice QCD determination of hyperon-nucleon scattering. One s-wave phase shift was determined for $n \Lambda$ scattering in both spin-channels at pion masses of 350,490 , and $590 \mathrm{MeV}$, and for $n \Sigma^{-}$scattering in both spin channels at pion masses of 490, and $590 \mathrm{MeV}$. The calculations were performed with domain-wall valence quarks on dynamical, staggered gauge configurations with a lattice spacing of $b \sim 0.125 \mathrm{fm}$.
\end{abstract}




\section{A. Introduction}

In high-density baryonic systems, the large value of the Fermi energy may make it energetically advantageous for some of the nucleons to become hyperons, with the increase in rest mass being more than compensated for by the decrease in Fermi energy. This is speculated to occur in the interior of neutron stars, but a quantitative understanding of this phenomenon depends on knowledge of the interactions among the hadrons in the medium. In contrast to nucleon-nucleon (NN) interactions, where the wealth of experimental data has allowed for the construction of high-precision potentials, the hyperon-nucleon (YN) interactions are only very-approximately known. Experimental information about the YN interaction comes mainly from the study of hypernuclei [1, 2], the analysis of associated $\Lambda$-kaon and $\Sigma$-kaon production in NN collisions near threshold [3, 4, ,5, 6, 7, 8], and hadronic atoms [9]. There are a total of 35 cross-sections measurements [10] of the processes $\Lambda p \rightarrow \Lambda p, \Sigma^{-} p \rightarrow \Lambda n$, $\Sigma^{+} p \rightarrow \Sigma^{+} p, \Sigma^{-} p \rightarrow \Sigma^{-} p$ and $\Sigma^{-} p \rightarrow \Sigma^{0} n$, and unsurprisingly, the extracted scattering parameters are highly model dependent. The theoretical study of YN interactions is hindered by the lack of experimental guidance. The "realistic" potentials developed by the Nijmegen [11, 12] and Jülich [13, 14, 15] groups are just two examples of phenomenological models based on meson exchange. These are soft-core potentials with one-boson exchange models of the NN interaction. Since $S U(3)$ flavor symmetry is broken by the differences in the quark masses, the corresponding couplings are not completely determined by the NN interaction and are instead obtained by a fit to the available data. In Ref. [11, 12], for example, six different models are constructed, each describing the available YN cross-section data equally well, but predicting different values for the phase shifts. The effective field theory approach [16, 17, 18, 19, 20] is less developed and suffers from a large number of couplings that need to be fit to the data.

In view of the large uncertainties in the YN scattering amplitudes and their importance for modeling neutron stars and the study of hypernuclei, a first-principles QCD calculation of YN scattering is highly desirable. The only way to achieve this is through numerical calculations using lattice QCD. In a previous paper [19], some of the present authors outlined a program to address this issue with a combination of lattice calculations and the use of effective field theories. This paper reports on the first results of the implementation of this program. In particular, we compute low-energy s-wave phase shifts for YN scattering in the ${ }^{1} S_{0}$ channel and ${ }^{3} S_{1}-{ }^{3} D_{1}$ coupled-channels at particular energies, using Lüscher's finitevolume method [21, 22, 23]. This is a straightforward extension of work by some of the present authors on NN scattering from lattice QCD [24]. The calculations were performed in the isospin limit on the coarse MILC lattices [25] for pions with masses of $\sim 290 \mathrm{MeV}$, $\sim 350 \mathrm{MeV}, \sim 490 \mathrm{MeV}$ and $\sim 590 \mathrm{MeV}$. However, we do not attempt to extrapolate to the physical pion mass as it is likely that all but one of the data points lies outside the regime of applicability of the YN EFT's.

\section{B. Methodology and Details of the Calculation}

Lattice QCD calculations of the interactions among hadrons are notoriously difficult and require circumventing the Maiani-Testa theorem [26], which states that one cannot compute Green's functions at infinite volume on the lattice and recover S-matrix elements except at kinematic thresholds. The s-wave scattering amplitude for two particles below inelastic thresholds can be determined using Lüscher's method [21, 22, 23], which entails a measure- 
ment of one or more energy levels of the two-particle system in a finite volume. Our computation uses the mixed-action lattice QCD scheme developed by LHPC [27, 28] which places domain-wall valence quarks from a smeared-source on $N_{f}=2+1$ asqtad-improved [29, 30] MILC configurations generated with rooted staggered sea quarks [25] that are hypercubicsmeared (HYP-smeared) [31, 32, 33, 34]. In the generation of the MILC configurations, the strange-quark mass was fixed near its physical value, $b m_{s}=0.050$, (where $b=0.125 \mathrm{fm}$ is the lattice spacing) determined by the mass of hadrons containing strange quarks. The two light quarks in the configurations are degenerate (isospin-symmetric), with masses $b m_{l}=0.007$, 0.010, 0.020 and 0.030. As was shown by LHPC [27, 28], HYP-smearing allows for a significant reduction in the residual chiral symmetry breaking at a moderate extent $L_{s}=16$ of the extra dimension and domain-wall height $M_{5}=1.7$. Using Dirichlet boundary conditions we reduced the original time extent of 64 down to 32. This allowed us to recycle propagators computed for the nucleon structure function calculations performed by LHPC. For bare domain-wall fermion masses we used the tuned values that match the staggered Goldstone pion to few-percent precision [27, 28]. The parameters used in the propagator calculation can be found in Ref. [35]. All propagator calculations were performed using the Chroma software suite [36, 37]. Eight propagators per configuration were computed at distinct source points on the lattice.

We found that the cleanest method for extracting the energy-difference between the YN state, and the mass of an isolated nucleon and an isolated hyperon, was by forming the ratio of correlation functions

$$
G_{Y N}^{S}(t)=C_{Y N}^{S}(t) /\left(C_{Y}(t) C_{N}(t)\right) \rightarrow e^{-\Delta E_{Y N} t}
$$

where $S$ denotes spin. At large times, this ratio depends exponentially upon the ground-state energy shift of the YN system due to interactions. The single nucleon correlator is

$$
C_{N}(t)=\sum_{\mathbf{x}}\left\langle N(t, \mathbf{x}) N^{\dagger}(0, \mathbf{0})\right\rangle
$$

and the single hyperon correlator has an analogous form. The YN correlator that projects onto the s-wave state in the continuum limit is

$$
\begin{aligned}
& C_{Y N}^{S}(t)=X_{\alpha \beta \sigma \rho}^{i j k l} \\
& \quad \sum_{\mathbf{x}, \mathbf{y}}\left\langle Y_{i}^{\alpha}(t, \mathbf{x}) N_{j}^{\beta}(t, \mathbf{y}) Y_{k}^{\sigma \dagger}(0, \mathbf{0}) N_{l}^{\rho \dagger}(0, \mathbf{0})\right\rangle,
\end{aligned}
$$

where $\alpha, \beta, \sigma, \rho$ are isospin-indices and $i, j, k, l$ are Dirac-indices. The tensor $X_{\alpha \beta \sigma \rho}^{i j k l}$ has elements that produce the correct spin-isospin quantum numbers for a hyperon and nucleon in an s-wave. The summation over $\mathbf{x}$ (and $\mathbf{y}$ ) corresponds to summing over all the spatial lattice sites, thereby projecting onto the momentum $\mathbf{p}=\mathbf{0}$ state of each particle seperately. The interpolating field for the proton is $p_{i}(t, \mathbf{x})=\epsilon_{a b c} u_{i}^{a}(t, \mathbf{x})\left(u^{b T}(t, \mathbf{x}) C \gamma_{5} d^{c}(t, \mathbf{x})\right)$, and similarly for the neutron and hyperons. We have used an interpolating field $n \times \Sigma^{-}$to determine the energy-eigenvalues of the s-wave strangeness $=1$, isospin $=\frac{3}{2}$ eigenstates in both spin channels, and an interpolating field $n \times \Lambda$ to determine the energy-eigenvalues of the s-wave strangeness $=1$, isospin $=\frac{1}{2}$ eigenstates in both spin channels.

Once the energy shift due to the YN interactions has been computed, the real part of the inverse scattering amplitude is determined via the Lüscher formula [21, 22, 23]. To extract $p \cot \delta(p)$, where $\delta(p)$ is the phase shift, the magnitude of the center-of-mass momentum, 
$p$, is extracted from the energy shift, $\Delta E_{Y N}=\sqrt{p^{2}+M_{Y}^{2}}+\sqrt{p^{2}+M_{N}^{2}}-M_{Y}-M_{N}$, and inserted into:

$$
p \cot \delta(p)=\frac{1}{\pi L} \mathbf{S}\left(\frac{p L}{2 \pi}\right)
$$

which is valid below the inelastic threshold. The regulated three-dimensional sum is

$$
\mathbf{S}(\eta) \equiv \sum_{\mathbf{j}}^{|\mathbf{j}|<\Lambda} \frac{1}{|\mathbf{j}|^{2}-\eta^{2}}-4 \pi \Lambda
$$

where the summation is over all triplets of integers $\mathbf{j}$ such that $|\mathbf{j}|<\Lambda$ and the limit $\Lambda \rightarrow \infty$ is implicit.

\section{Results}

The effective mass plots of the ratio of correlation functions with identifiable plateaus obtained at $m_{\pi} \sim 350 \mathrm{MeV}, m_{\pi} \sim 490 \mathrm{MeV}$ and $m_{\pi} \sim 590 \mathrm{MeV}$ are shown in figs. 1, 2, 3 and 4 . Single and double exponential forms were fit to the correlation functions by
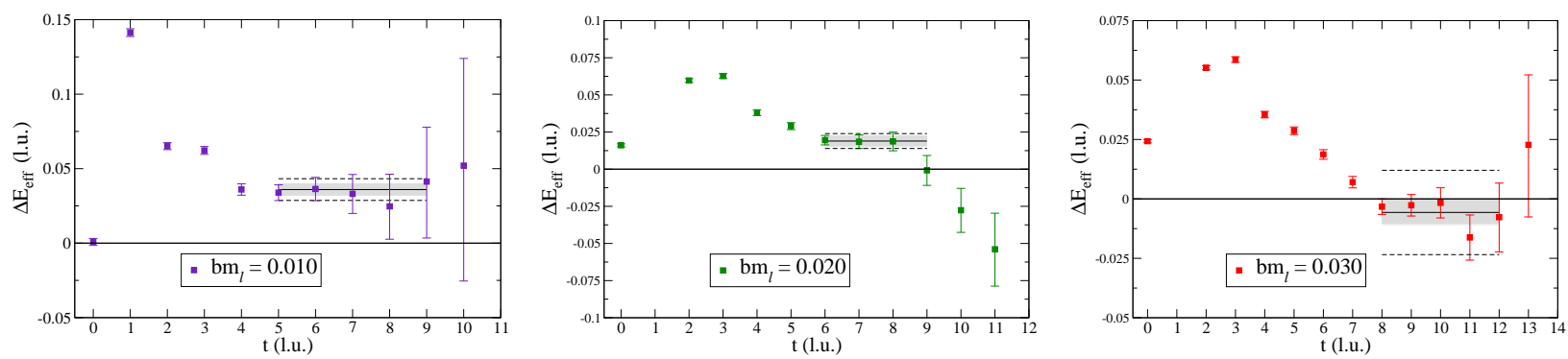

FIG. 1: Effective mass plots for $n \Lambda$ in the ${ }^{1} S_{0}$-channel at pion masses of $m_{\pi} \sim 350 \mathrm{MeV}$ (left panel), $m_{\pi} \sim 490 \mathrm{MeV}$ (center panel) and $m_{\pi} \sim 590 \mathrm{MeV}$ (right panel). The straight line and shaded region correspond to the extracted energy shift and associated uncertainty. The dashed lines correspond to the statistical and systematic errors added linearly.

$\chi^{2}$-minimization, from which the $Y N$ energy shifts were determined. The central values and statistical uncertainties were determined by the jackknife procedure over the ensemble of configurations, and are shown in Table \.

The plateaus in the correlator ratios $G_{Y N}(t)$ persist for only a small number of time-slices. At small $t$ there is the usual contamination from excited states whereas at larger $t$ the signalto-noise ratio degrades exponentially with $t$ according to $e^{-\left(M_{N}+M_{Y}-3 m_{\pi}\right) t}[38]$. The Dirichlet boundary at $t=22$ introduces a systematic uncertainty due to backward propagating states. However, in practice, the statistical noise becomes a limiting factor at far earlier time slices and the boundary at $t=22$ is not an issue for this calculation. We obtained a non-zero energy shift larger than the statistical error in ten of the $G_{Y N}$ correlation functions, as shown in Table I. The phase shifts $\delta$ (and $-1 / k \cot \delta(k)$ ) were determined through the Lüscher formula and their errors by the jackknife procedure (we do not give a value of $\delta$ for a negative energy shift). The quantities in Table\that are in physical units were obtained with a lattice 

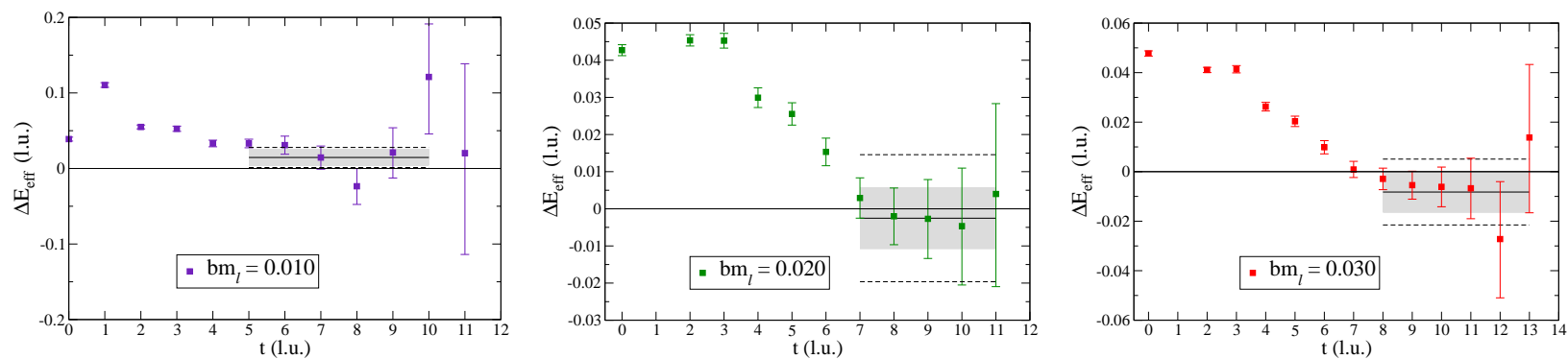

FIG. 2: Effective mass plots for $n \Lambda$ in the ${ }^{3} S_{1}$-channel at pion masses of $m_{\pi} \sim 350 \mathrm{MeV}$ (left panel), $m_{\pi} \sim 490 \mathrm{MeV}$ (center panel) and $m_{\pi} \sim 590 \mathrm{MeV}$ (right panel). The straight line and shaded region correspond to the extracted energy shift and associated uncertainty. The dashed lines correspond to the statistical and systematic errors added linearly.
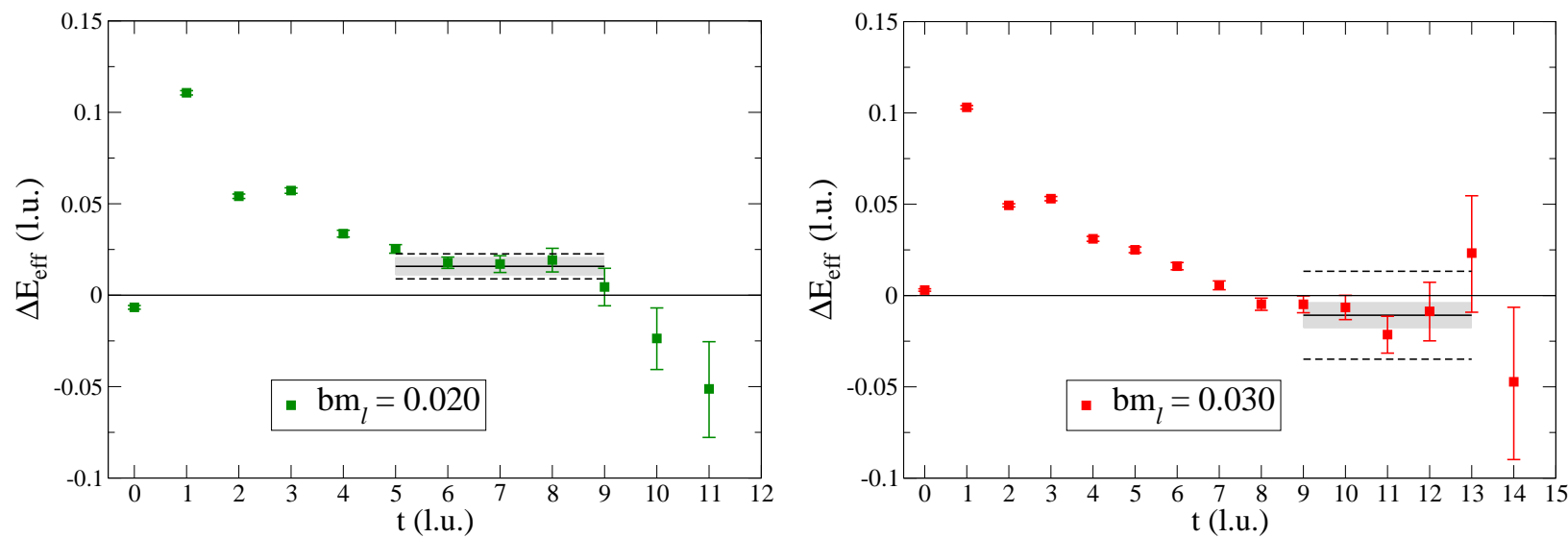

FIG. 3: Effective mass plots for $n \Sigma^{-}$in the ${ }^{1} S_{0}$-channel at pion masses of $m_{\pi} \sim 490 \mathrm{MeV}$ (left panel) and $m_{\pi} \sim 590 \mathrm{MeV}$ (right panel). The straight line and shaded region correspond to the extracted energy shift and associated uncertainty. The dashed lines correspond to the statistical and systematic errors added linearly.

spacing of $b=0.125 \mathrm{fm}$ set by MILC, which is consistent with the determination from [39] ( $b=0.1274 \pm 0.0007 \pm 0.0003 \mathrm{fm}$ ) based on the chiral expansion of the pion decay constant. We have not shown the results for channels in which there is no clear plateau in the effective mass plot. This is the case for all $m_{\pi} \sim 290 \mathrm{MeV}\left(m_{l}=0.007\right)$ correlation functions where the rapid decrease of the signal-to-noise ratio caused by the small pion mass eliminated all plateaus. The systematic errors shown in Table@are determined by varying the fitting range, and by comparing the results of fitting one and two exponentials to the ratio of correlation functions.

It is not clear that we have been able to identify the ground states in all of the correlation functions, e.g. $n \Sigma^{-}$in the ${ }^{1} S_{0}$-channel at $m_{\pi} \sim 490 \mathrm{MeV}$, and $n \Lambda$ in the ${ }^{1} S_{0}$-channel at $m_{\pi} \sim 490 \mathrm{MeV}$, as the statistics are not sufficient to determine whether the large-time behavior we observe is due to noise or due to the presence of any states with lower energy than those shown in Table II. Indeed, it would be very exciting if there were states with lower energy, as they would likely be bound states (based on naturalness arguments and the exact Lüscher relation). This uncertainty in no way undermines our results; regardless of the 

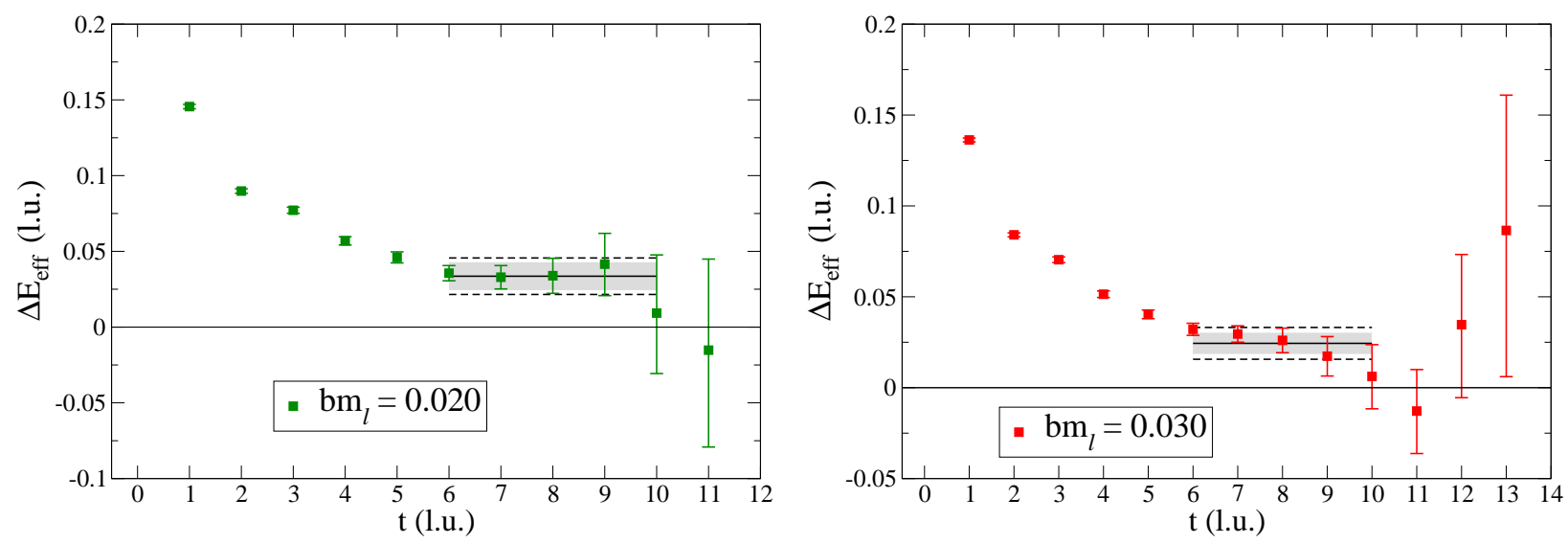

FIG. 4: Effective mass plots for $n \Sigma^{-}$in the ${ }^{3} S_{1}$-channel at pion masses of $m_{\pi} \sim 490 \mathrm{MeV}$ (left panel) and $m_{\pi} \sim 590 \mathrm{MeV}$ (right panel). The straight line and shaded region correspond to the extracted energy shift and associated uncertainty. The dashed lines correspond to the statistical and systematic errors added linearly.

\begin{tabular}{|c|c|c|c|c|c|c|}
\hline Channel & $m_{\pi}(\mathrm{MeV})$ & Range & $\Delta E(\mathrm{MeV})$ & $|\mathbf{k}|(\mathrm{MeV})$ & $\delta($ degrees $)$ & $-(k \cot \delta)^{-1}(\mathrm{fm})$ \\
\hline \hline$n \Lambda$ & $592 \pm 1 \pm 10$ & $8-12$ & $-9 \pm 8 \pm 20$ & - & - & $0.8 \pm 1.4 \pm 0.4$ \\
\cline { 2 - 7 } & $493 \pm 1 \pm 8$ & $6-9$ & $29.8 \pm 5.4 \pm 2.5$ & $197 \pm 24 \pm 4$ & $-32.3 \pm 8.1 \pm 2.8$ & $0.63 \pm 0.12 \pm 0.014$ \\
\cline { 2 - 7 } & $354 \pm 1 \pm 6$ & $5-9$ & $56.8 \pm 6.0 \pm 5.5$ & $255 \pm 22 \pm 13$ & $-53.4 \pm 8.5 \pm 10.1$ & $1.04 \pm 0.24 \pm 0.15$ \\
\hline \hline \multirow{2}{*}{$n \Lambda$} & $592 \pm 1 \pm 10$ & $8-13$ & $-13 \pm 13 \pm 8$ & - & - & $3 \pm 14 \pm 2$ \\
\cline { 2 - 7 } & $493 \pm 1 \pm 8$ & $7-11$ & $-4 \pm 13 \pm 14$ & - & - & $(-\infty, \infty)$ \\
\cline { 2 - 7 } & $354 \pm 1 \pm 6$ & $5-10$ & $23 \pm 17 \pm 4$ & $168 \pm 62 \pm 14$ & $-23 \pm 18 \pm 4$ & $0.50 \pm 0.26 \pm 0.06$ \\
\hline \hline \multirow{2}{*}{$n \Sigma^{-}$} & $592 \pm 1 \pm 10$ & $9-13$ & $-17 \pm 11 \pm 27$ & - & - & $(-\infty, \infty)$ \\
\cline { 2 - 7 } & $493 \pm 1 \pm 8$ & $5-9$ & $24.9 \pm 7.8 \pm 3.0$ & $179 \pm 28 \pm 11$ & $-27.2 \pm 9.0 \pm 3.8$ & $0.57 \pm 0.13 \pm 0.05$ \\
\hline \hline${ }^{1} S_{0}$ & $592 \pm 1 \pm 10$ & $6-10$ & $38.5 \pm 8.8 \pm 5.0$ & $226 \pm 26 \pm 15$ & $-44.3 \pm 9.8 \pm 5.4$ & $0.85 \pm 0.20 \pm 0.10$ \\
\cline { 2 - 7 } & $493 \pm 1 \pm 8$ & $6-10$ & $53 \pm 14 \pm 5$ & $261 \pm 35 \pm 13$ & $-58 \pm 15 \pm 5$ & $1.19 \pm 0.51 \pm 0.15$ \\
\hline
\end{tabular}

TABLE I: Summary of results from the $G_{Y N}$ correlation functions which exhibit a clear plateau in the effective energy plot. The first error is statistical and the second error is systematic.

nature of the states shown in Table \, they are clearly YN states present in the continuum.

In addition to the fitting systematics given in Table I there are other systematic uncertainties in our calculations that we have not shown, as they are all expected to be small in comparison. The discretization errors due to the finite lattice spacing arising from the sea-action are of order $\mathcal{O}\left(\alpha_{s} b^{2}\right)$ ( $\alpha_{s}$ is the strong coupling constant) and those in the valence sector of order $\mathcal{O}\left(b^{2}\right)$ due to the near-perfect chiral symmetry. The agreement between continuum chiral perturbation theory and other results based on the same discretization scheme [24, 39, 43, 44] strongly suggest that the discretization errors in the hyperon interactions are, at most, of the order of a few percent. This is much smaller than the statistical errors quoted in Table I. A similar point can be made regarding the use of different fermion actions in the valence and sea sectors. Further, the relation between energy levels and phase shifts, eq. (4), is valid only up to corrections that are exponentially small in the volume. The 
corrections to the Lüscher formula can be computed in chiral perturbation theory, as shown in the $\pi \pi$ case in [41] and for two nucleons in [42]. These effects are particularly small in the $N \Lambda$ system, as the long-range part of the interaction is dominated by two-pion exchange and one-kaon exchange, and not one-pion exchange.
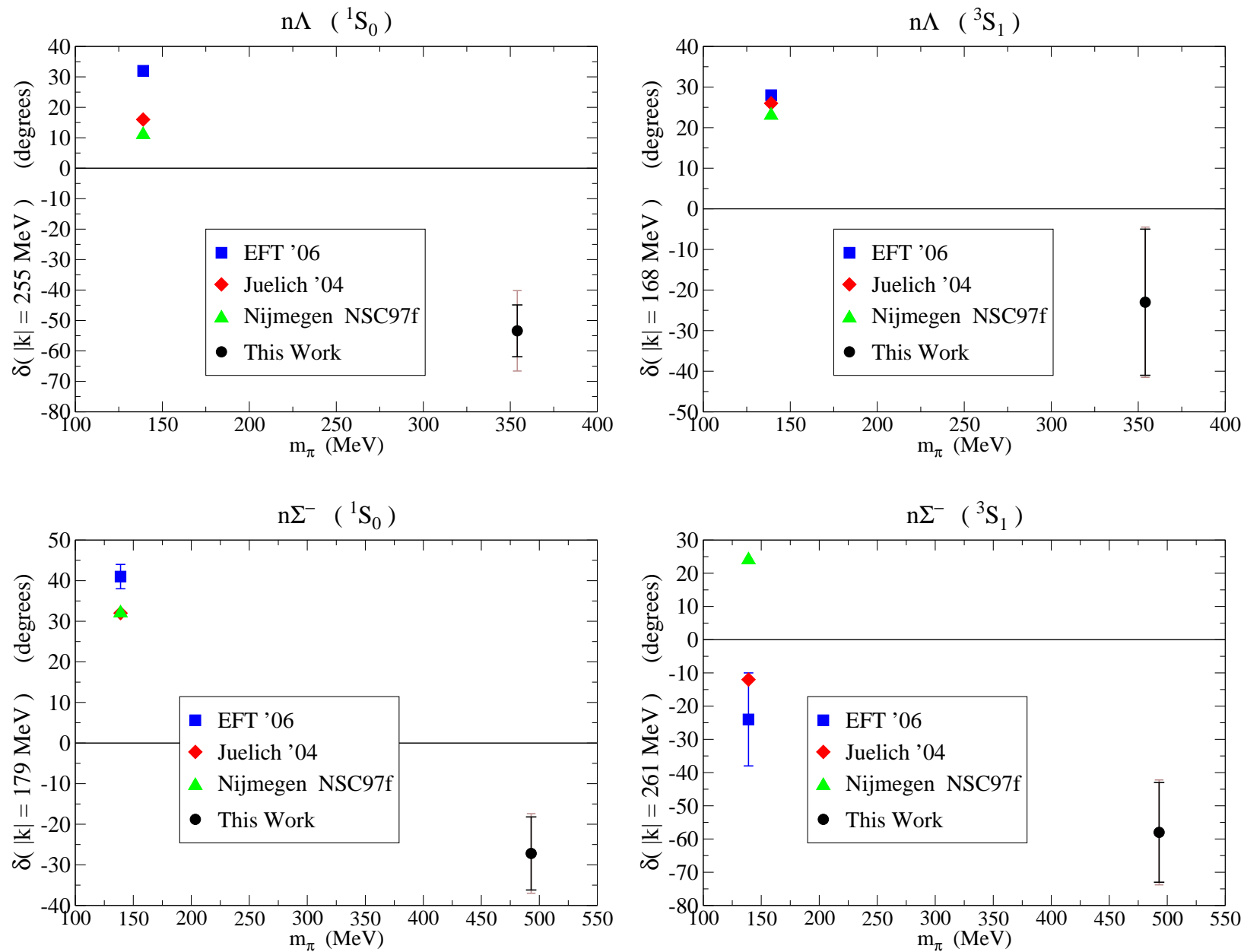

FIG. 5: Comparison of the lowest-pion-mass lattice results in each channel with a recently developed YN EFT [20] (squares), and several potential models: Nijmegen [11] (triangles) and Jülich [15] (diamonds). The dark error bars on the lattice data are statistical and the light error bars are statistical and systematic errors added in quadrature.

\section{Discussion}

We have presented results of the first fully-dynamical lattice QCD calculation of YN interactions. The scattering amplitudes for s-wave $n \Lambda$ and $n \Sigma^{-}$, in both the ${ }^{1} S_{0^{-}}$channel and the ${ }^{3} S_{1}-{ }^{3} D_{1}$ coupled-channels, have been determined at one value of momentum for pion masses of $\sim 350 \mathrm{MeV}, \sim 490 \mathrm{MeV}$ and $590 \mathrm{MeV}$. Unfortunately, the lightest pion mass at which we have been able to extract a signal is at the upper limits of the regime of applicability of the effective field theories that have been constructed, thus precluding a chiral extrapolation. However, this work does provide new rigorous theoretical constraints on effective 
field theory, and potential model constructions of YN interactions. In fig. 5 we compare the lattice values of the phase shifts to recent EFT results [20] (squares), and to several potential models: Nijmegen [11] (triangles) and Jülich [15]. At face value these results appear quite discrepant, however one should keep in mind that extrapolation to the physical pion mass will seriously alter individual contributions to the $\mathrm{YN}$ interaction.

While the measurements of the momenta and phase shifts are unambiguous, their physical interpretation is not entirely resolved. Each of the phase shifts at the lowest pion masses are negative. Assuming that the observed state is the ground state in the lattice volume, this implies that the interactions are all repulsive. The $n \Sigma^{-}$interaction in the ${ }^{3} S_{1}-{ }^{3} D_{1}$ coupled channels is strongly repulsive at $m_{\pi} \sim 490 \mathrm{MeV}$, while the interaction in the ${ }^{1} S_{0}$-channel is only mildly repulsive. The opposite is found to be true for the $n \Lambda$ systems at $m_{\pi} \sim 350 \mathrm{MeV}$, where the interaction in the ${ }^{1} S_{0}$-channel is found to be strongly repulsive, while that in the ${ }^{3} S_{1}-{ }^{3} D_{1}$ coupled channels is mildly repulsive. However, there may be channels for which there exist states of lower, negative energies, some of which may correspond to bound states in the continuum limit. If such states are present, then we would conclude that the interaction is attractive, and that the positive-shifted energy state we have identified corresponds to the first continuum level. Current statistics are sufficiently poor that nothing definitive can be said about the existence of such states. Therefore, we are continuing to accumulate statistics and experiment with signal optimization in order to resolve this issue.

It is clear that a precise lattice QCD calculation of YN scattering will have dramatic impact upon the field of hypernuclear physics, and may have an equal impact on our ability to determine the evolution of neutron stars, simply due to the present absence of precise experimental data. We have performed the first of such calculations, albeit at unphysically large pion masses. The present work was limited entirely by the lack of computational resources. We hope that this limitation recedes in the future, and that lattice QCD can be developed as a reliable tool to calculate the interactions between baryons in experimentally inaccessible or difficult areas of strong interactions.

\section{E. Acknowledgments}

We thank R. Edwards for help with the QDP++/Chroma programming environment [36] with which the calculations discussed here were performed. The computations for this work were performed at Jefferson Lab, Fermilab, Lawrence Livermore National Laboratory, National Center for Supercomputing Applications, Centro Nacional de Supercomputación (Barcelona, Spain) and ASTRON-BlueGene/L at the Reken Centrum of Groningen University. We are indebted to the MILC and the LHP collaborations for use of their configurations and propagators, respectively. The work of MJS was supported in part by the U.S. Dept. of Energy under Grant No. DE-FG03-97ER4014. The work of KO was supported in part by the U.S. Dept. of Energy contract No. DE-AC05-06OR23177 (JSA) and contract No. DEAC05-84150 (SURA). The work of PFB was supported in part by the U.S. Dept. of Energy grant No. ER-40762-365. The work of SRB was supported in part by the National Science Foundation under grant No. PHY-0400231. Part of this work was performed under the auspices of the US DOE by the University of California, Lawrence Livermore National Laboratory under Contract No. W-7405-Eng-48. AP is supported by the Ministerio de Educación y Ciencia (Spain) under contract No. FIS2005-03142 and by the Generalitat de 
Catalunya under contract No. 2005SGR-00343.

[1] A. Gal, E. Hungerford, Nucl. Phys. A 754 1-489 (2005).

[2] O. Hashimoto and H. Tamura, Prog. Part. Nucl. Phys. 57, 564 (2006).

[3] J. Balewski et al., Phys. Lett. B 420, 211 (1998).

[4] S. Sewerin et al., Phys. Rev. Lett. 83, 682 (1999).

[5] P. Kowina et al., Eur. Phys. j. A22, 293 (2004).

[6] R. Bilger et al., Phys. Lett. B 420, 217 (1998).

[7] M. Abdel-Bary et al., Phys. Lett. B 595, 127 (2004).

[8] A. Gasparyan, J. Haidenbauer, C. Hanhart, J. Speth, Phys. Rev. C 69, 034006 (2004).

[9] C. J. Batty, E. Friedman and A. Gal, Phys. Rept. 287 (1997) 385.

[10] http://nn-online.sci.kun.nl/index.html.

[11] V.G.J. Stoks and Th.A. Rijken, Phys. Rev. C 59, 3009 (1999); Th.A. Rijken, V.G.J. Stoks and Y. Yamamoto, Phys. Rev. C 59, 21-40 (1999).

[12] Th.A. Rijken, Y. Yamamoto, Phys. Rev. C 73044008 (2006).

[13] B. Holzenkamp, K. Holinde, and J. Speth, Nucl. Phys. A 500 (1989) 485.

[14] A. Reuber, K. Holinde, H.-C. Kim, and J. Speth, Nucl. Phys. A 608243 (1996).

[15] J. Haidenbauer and Ulf G. Meißner, Phys. Rev. C 72044005 (2005).

[16] M. J. Savage and M. B. Wise, Phys. Rev. D 53, 349 (1996).

[17] C.L. Korpa, A.E.L. Dieperink, and R.G.E. Timmermans, Phys. Rev. C 65, 015208 (2001).

[18] H.W. Hammer, Nucl. Phys. A 705, 173 (2002).

[19] S.R. Beane, P.F. Bedaque, A. Parreño, M.J. Savage, Nucl. Phys. A 747, 55 (2005).

[20] H. Polinder, J. Haidenbauer and Ulf-G. Meißner, Nucl. Phys. A 779, 244 (2006) arXiv:nucl-th/0605050.

[21] H. W. Hamber, E. Marinari, G. Parisi and C. Rebbi, Nucl. Phys. B 225, 475 (1983),

[22] M. Lüscher, Nucl. Phys. B 354, 531 (1991).

[23] S. R. Beane, P. F. Bedaque, A. Parreno and M. J. Savage, Phys. Lett. B 585, 106 (2004) arXiv:hep-lat/0312004].

[24] S. R. Beane, P. F. Bedaque, K. Orginos and M. J. Savage, Phys. Rev. Lett. 97, 012001 (2006).

[25] C. W. Bernard et al., Phys. Rev. D 64, 054506 (2001).

[26] L. Maiani and M. Testa, Phys. Lett. B 245, 585 (1990).

[27] D. B. Renner et al., Nucl. Phys. Proc. Suppl. 140, 255 (2005).

[28] R. G. Edwards et al., PoS LAT2005, 056 (2005).

[29] K. Orginos, D. Toussaint and R. L. Sugar, Phys. Rev. D 60, 054503 (1999).

[30] K. Orginos and D. Toussaint, Phys. Rev. D 59, 014501 (1999).

[31] A. Hasenfratz and F. Knechtli, Phys. Rev. D 64, 034504 (2001).

[32] T. A. DeGrand, A. Hasenfratz and T. G. Kovacs, Phys. Rev. D 67, 054501 (2003).

[33] T. A. DeGrand, Phys. Rev. D 69, 014504 (2004).

[34] S. Dürr, C. Hoelbling and U. Wenger, Phys. Rev. D 70, 094502 (2004).

[35] S. R. Beane, P. F. Bedaque, T. C. Luu, K. Orginos, E. Pallante, A. Parreno and M. J. Savage, Phys. Rev. D 74, 114503 (2006), arXiv:hep-lat/0607036.

[36] R. G. Edwards and B. Joo, Nucl. Phys. Proc. Suppl. 140 (2005) 832.

[37] C. McClendon, Jlab preprint, JLAB-THY-01-29 (2001).

[38] G. P. Lepage, CLNS-89-971 Invited lectures given at TASI'89 Summer School, Boulder, CO, 
Jun 4-30, 1989

[39] S. R. Beane, P. F. Bedaque, K. Orginos and M. J. Savage, Phys. Rev. D 73, 054503 (2006).

[40] F. D. R. Bonnet, R. G. Edwards, G. T. Fleming, R. Lewis and D. G. Richards, Phys. Rev. D 72, 054506 (2005), C. Alexandrou, S. Gusken, F. Jegerlehner, K. Schilling and R. Sommer, Nucl. Phys. B 414, 815 (1994).

[41] P. F. Bedaque, I. Sato and A. Walker-Loud, Phys. Rev. D 73, 074501 (2006).

[42] P. F. Bedaque and I. Sato, to appear.

[43] S. R. Beane, K. Orginos and M. J. Savage, arXiv:hep-lat/0604013.

[44] S. R. Beane, K. Orginos and M. J. Savage, arXiv:hep-lat/0605014. 